\title{
Europium, Samarium, and Neodymium Isotopic Fractions in Metal-Poor Stars
}

\author{
Ian U. Roederer*, James E. Lawler ${ }^{\dagger}$, Christopher Sneden*, John J. Cowan**, \\ Jennifer S. Sobeck*, and Catherine A. Pilachowski ${ }^{\S}$ \\ ${ }^{*}$ Department of Astronomy, University of Texas at Austin, 1 University Station, C1400, Austin, TX $78712-0259$ \\ ${ }^{\dagger}$ Department of Physics, University of Wisconsin, 1150 University Avenue, Madison, WI 53706 \\ ${ }^{* *}$ Department of Physics and Astronomy, University of Oklahoma, Room 131, Nielsen Hall, Norman, OK 73019 \\ *European Southern Observatory, Karl-Schwarzschild-Strasse 2, D-85748 Garching bei München, Germany \\ $\S$ Department of Astronomy, Indiana University, Swain West 319, 727 E. Third St., Bloomington, IN 47405-7105
}

\begin{abstract}
We have derived isotopic fractions of europium (Eu), samarium (Sm), and neodymium (Nd) in two metal-poor giants with differing neutron-capture nucleosynthetic histories. These isotopic fractions were measured from new very high resolution $(R \sim 120,000)$, high signal-to-noise $(\mathrm{S} / \mathrm{N} \sim 160-1000)$ spectra obtained with the 2dCoudé spectrograph of McDonald Observatory's $2.7 \mathrm{~m}$ Smith telescope. Synthetic spectra were generated using recent high-precision laboratory measurements of hyperfine and isotopic subcomponents of several transitions of these elements and matched quantitatively to the observed spectra. We interpret our isotopic fractions by the nucleosynthesis predictions of the stellar model, which models $s$-process nucleosynthesis in the physical conditions expected in a low-mass, thermally-pulsing star on the AGB, and the classical method, which assumes that $s$-process nucleosynthesis can be approximated by a steady neutron flux impinging upon Fe-peak seed nuclei. These two approaches predict the relative contributions to the Solar System $n$-capture abundances from the $s$ - and $r$-processes and, by extension, the relative contributions of these two process to material in metal-poor stars. Our Eu isotopic fraction in $\mathrm{HD} 175305$ is consistent with an $r$-process origin by the classical method and is consistent with both an $r$-process and $s$-process origin by the stellar model. Our Sm isotopic fraction in $\mathrm{HD} 175305$ is consistent with a predominantly $r$-process origin by both methods, and our Sm isotopic fraction in HD 196944 is consistent with a pure $s$-process origin by both methods as well. Our $\mathrm{Nd}$ isotopic fractions in both stars are consistent with either $r$-process and $s$-process origins by both methods. The Eu and Sm isotopic fraction estimates argue for an $r$-process origin for the rare-earth elements in HD 175305 and an $s$-process origin for them in HD 196944, in excellent agreement with previous studies of the elemental abundance distributions in these stars. This study for the first time extends the $n$-capture origin of multiple rare earths in metal-poor stars from elemental abundances to the isotopic level, strengthening the $r$-process interpretation for HD 175305 and the $s$-process interpretation for $\mathrm{HD} 196944$.
\end{abstract}

Keywords: atomic data; nuclear reactions; nucleosynthesis; abundances; stars: abundances; stars: Population II PACS: $97.10 . \mathrm{Cv} ; 97.10 . \mathrm{Ex} ; 97.10 . \mathrm{Tk} ; 97.20 . \mathrm{Tr}$

\section{INTRODUCTION}

Nuclides with $Z>30$ are produced by stars through either the rapid (r)-process or the slow (s)-process. Two methods can be used to decompose the Solar System (S. S.) isotopic abundances into their constituent $s$ - and $r$-process origins-the "standard" or "classical" method [e.g., 1, 2, 3, 4] and the "stellar" model [5]. The classical method is model-independent, but it assumes an empirical, smoothly-varying relationship for the abundance curve and ignores details of the nuclear physics. The stellar method does not depend on knowledge of the S. S. abundance distribution, but is heavily dependent on nuclear physics laboratory measurements and complex stellar asymptotic giant branch (AGB) model calculations. Isotopic abundances should be more fundamental indicators of neutron (n)-capture nucleosynthesis than elemental abundances because they can directly confront $r$-process and $s$-process predictions without the smearing effect of multiple isotopes. The wavelength of a spectral line is split by two effects, hyperfine structure (HFS) and isotope shifts, and the isotopic fractions can be measured by detailed comparisons of an observed absorption line profile to synthetic spectra of these line substructures. A handful of previous studies have quantitatively examined the isotopic abundances of $\mathrm{Ba}[6,7,8]$ and $\mathrm{Eu}[9,10,11]$ in metal-poor stars, and recently $\mathrm{Sm}$ has been qualitatively investigated as well [12]; no measurements of the $\mathrm{Nd}$ isotopic fraction have been made outside of the S. S., and no study has yet attempted to measure isotopic fractions of multiple $n$-capture elements in the same star.

\section{OBSERVATIONS AND ANALYSIS}

We choose two bright, well-studied, metal-poor giants for our analysis, HD 175305 and HD 196944. The elemental abundance distributions of HD 175305 and 
HD 196944 suggest an $r$-process and an $s$-process origin, respectively. We acquired new observations of these stars using the 2dCoudé cross-dispersed échelle spectrograph on the $2.7 \mathrm{~m}$ Harlan J. Smith Telescope at the W. J. McDonald Observatory. Our data have FWHM resolving powers $R \sim 120,000$, covering $4120 \leq \lambda \leq 5890 \AA$ in HD 175305 and $4200 \leq \lambda \leq 6640 \AA$ in HD 196944 ; however, even when using multiple grating settings our wavelength coverage is still incomplete within these ranges. Image processing, order extraction, and wavelength calibration were performed using standard IRAF packages. Our final $\mathrm{S} / \mathrm{N}$ values (per pixel) range from $\sim 160$ at $4130 \AA$ to $\sim 330$ at $5100 \AA$ in $\mathrm{HD} 175305$ and $\sim 160$ at $4200 \AA$ to $\sim 400$ at $5000 \AA$ in HD 196944 .

We re-derive atmospheric parameters for our stars that are in good agreement with many previous studies $\left(T_{\text {eff }} / \log (g) /[\mathrm{Fe} / \mathrm{H}] / v_{t}=4870 / 2.15 /-1.60 / 1.2\right.$ for HD 175305 and 5170/1.80/-2.46/1.7 for HD 196944). We collect the isotopes of $\mathrm{Nd}$ ( 7 naturally-occurring isotopes), $\mathrm{Sm}$ (7 isotopes), and $\mathrm{Eu}$ (2 isotopes) into the isotopic fractions shown in Tab. 1. Eu HFS and isotope shifts are taken from previous studies [13, 14]. We compute the full HFS and isotope shift patterns for $\mathrm{Nd}$ and $\mathrm{Sm}$ using the recent laboratory measurements [Nd: 15, 16] and [Sm: 12, 17, 18]. Synthetic spectra are computed with the LTE spectrum analysis code MOOG [19].

The isotopic fractions can be measured by using three methods. First, the shape of the synthetic line profile can be compared with the observations using a $\chi^{2}$ fitting algorithm; this method proved most useful for the $\mathrm{Eu}$ and Sm lines. Second, the absolute wavelengths of the observed and synthetic spectra can by matched using wavelength standard Fe I $[20,21]$ and Ti II $[22,23]$ lines, and then the peak wavelength of the rare-earth line of interest can be measured. When matching multiple (i.e., 1-6) wavelength standards in the same échelle order, the scatter in our measured wavelength offsets necessary to ensure the matching of each line is $\sim 5 \mathrm{~m} \AA$, much greater than anticipated and not precise enough to enable the isotopic fraction to be measured. Unable to identify and quantify the source of the discrepancy between our wavelength standards, we are unable to proceed with confidence by this method of analysis. The third method matches the observed and synthetic spectra of each line by using the point in the synthetic spectrum that is insensitive to the $s$ - or $r$-process mix; this method is the only way we have been able to reliably assess the $\mathrm{Nd}$ isotopic fraction.

\section{RESULTS AND INTERPRETATION}

In $\mathrm{HD} 175305$, our $\mathrm{Sm}$ isotopic fraction $f_{152+154}=$ $0.51 \pm 0.08$, measured from profile fits to the 4424 and

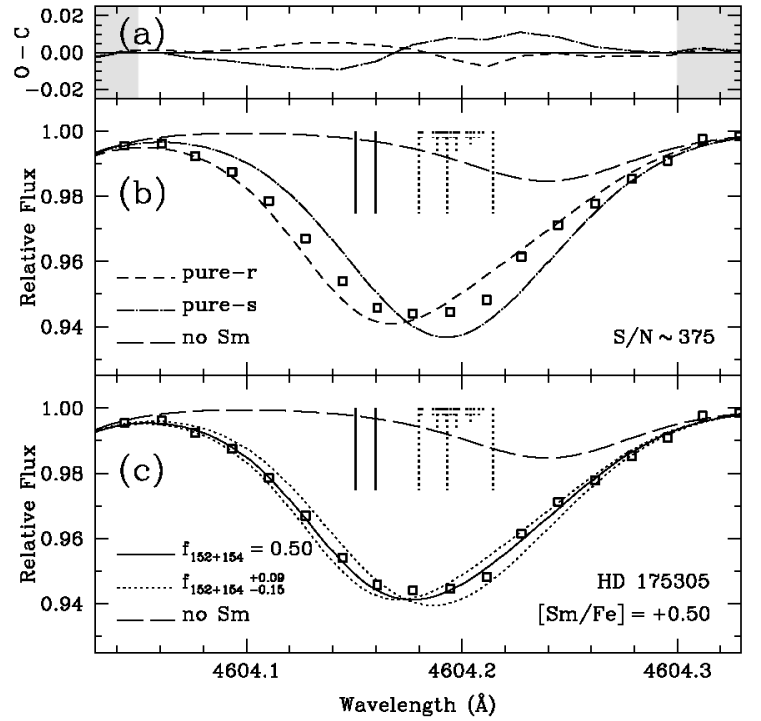

FIGURE 1. Our synthesis of the Sm II $4604 \AA$ line in $\mathrm{HD}$ 175305. The observed spectrum is indicated by open squares. In panel a, we show an $(O-C)$ plot for the pure-sand pure- $r$-process syntheses shown in panel $\mathbf{b}$. The unshaded region indicates the wavelengths over which we measure the isotopic fraction using the $\chi^{2}$ algorithm. In panel $\mathbf{b}$, we show syntheses for the pure- $s$ - and pure- $r$-process syntheses (the dotdashed and short-dashed curves, respectively) illustrating the contrast between these extremes. In panel c, we show the synthesis of our best fit, represented by the solid curve, and the $3 \sigma$ uncertainties, represented by the dotted curves. The longdashed curve is a synthesis with no Sm present, indicating the presence of blending features. The vertical sticks represent the positions and relative strengths of the individual hyperfine components; solid sticks represent the two heaviest isotopes, while dashed sticks represent the lighter five isotopes. We measure a Sm isotopic fraction of $f_{152+154}=0.50_{-0.15}^{+0.09}$ from this line, which suggests an $r$-process origin.

$4604 \AA$ lines (see Fig. 1), suggests an $r$-process origin. It is tempting to surmise that our $\mathrm{Nd}$ isotopic fraction, $f_{142+144}=0.21_{-0.21}^{+0.56}$, derived from three lines, is suggestive of an $r$-process origin, but the large uncertainty cannot exclude an $s$-process origin. Our Eu isotopic fraction, $f_{151}=0.50 \pm 0.04$, derived from only the $4129 \AA$ line and shown to be a reasonable match for the 4205 and $4435 \AA$ lines, implies an $r$-process origin by the classical method predictions but excludes neither a pure-s- nor a pure- $r$-process origin by the stellar model predictions. Our isotopic fractions in HD 175305 suggest a nucleosynthetic history primarily dominated by the $r$-process. The agreement between the elemental and isotopic abundance distributions in HD 175305 add to the preponderance of evidence (e.g., the growing number of $r$-processenhanced stars that conform to the scaled-S. S. $r$-process elemental abundance distribution) that supports the hy- 
TABLE 1. Isotope Mixes for Pure $s$ - and $r$-process Material and Measured Isotopic Fractions

\begin{tabular}{cccccccc}
\hline & \multicolumn{2}{c}{ Stellar Model } & \multicolumn{2}{c}{ Classical Method } & & & \\
Species & $s$-only & $r$-only & $s$-only & $r$-only & S. S. & HD 175305 & HD 196944 \\
\hline Nd: $f_{142+144}=$ & 0.67 & 0.32 & 0.69 & 0.27 & 0.510 & $0.21_{-0.21}^{+0.56}$ & $0.36_{-0.36}^{+0.64}$ \\
Sm: $f_{152+154}=$ & 0.21 & 0.64 & 0.20 & 0.64 & 0.495 & $0.51 \pm 0.08$ & $0.35 \pm 0.14$ \\
Eu: $f_{151}=$ & 0.54 & 0.47 & 0.04 & 0.47 & 0.478 & $0.50 \pm 0.04$ & \\
\hline
\end{tabular}
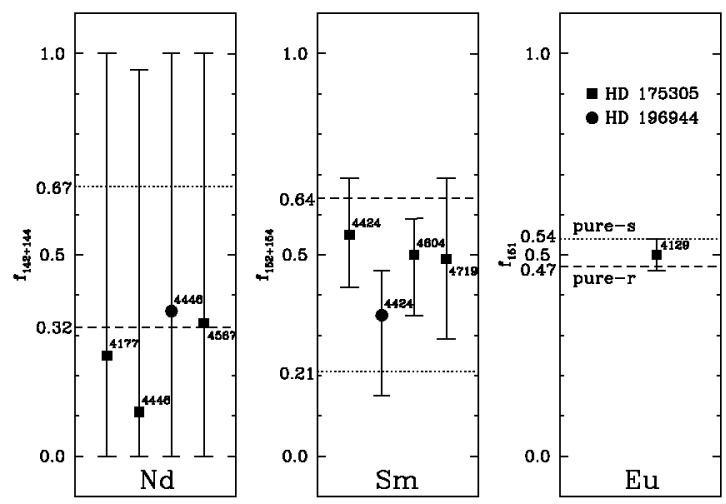

FIGURE 2. A summary of our best isotopic fraction measurements for each species. The Nd isotopic fractions reported here were measured by the method of matching the observed and synthetic spectra at the point of insensitivity to the isotopic mix. The Sm and Eu isotopic fractions were measured by fitting the shape of the line profile. Squares represent measurements in HD 175305, while circles represent measurements in HD 196944. The dotted line and dashed line represent the pure$s$ - and pure-r-process stellar model predictions.

pothesis of a universal $r$-process mechanism for elements with $Z \geq 56$.

In HD 196944, our measured Sm isotopic fraction, $f_{152+154}=0.35 \pm 0.14$, is suggestive of an $s$-process origin. Our Nd isotopic fraction, $f_{142+144}=0.36_{-0.36}^{+0.64}$ can exclude no possible values of the isotopic mix. The interpretation for the $\mathrm{Sm}$ is consistent with the $s$-process elemental abundance signatures and radial velocity variations observed for this star [24]. Our best measurements for each species are summarized in Fig. 2 and Tab. 1 for both HD 175305 and HD 196944.

\section{ACKNOWLEDGMENTS}

Funding for this project has been generously provided by the U. S. National Science Foundation (grant AST 0307279 to J. J. C., grant AST 05-06324 to J. E. L., and grants AST 03-07495 and AST 06-07708 to C. S.) and by the Sigma Xi Grants-in-Aid-of-Research program.

\section{REFERENCES}

1. Clayton, D. D., Fowler, W. A., Hull, T. E., \& Zimmermann, B. A. 1961, Ann. Phys., 12, 331

2. Käppeler, F., Beer, H., \& Wisshak, K. 1989, Rep. Prog. Phys., 52, 945

3. Cowan, J. J., Lawler, J. E., Sneden, C., Den Hartog, E. A., \& Collier, J. 2006, in Proc. NASA LAW, ed. V. Kwong, (Washington: NASA), 82

4. Seeger, P. A., Fowler, W. A., \& Clayton, D. D. 1965, ApJS, 11,121

5. Arlandini, C., Käppeler, F., Wisshak, K., Gallino, R., Lugaro, M., Busso, M., \& Straniero, O. 1999, ApJ, 525, 886

6. Magain, P. 1995, A\&A, 297, 686

7. Lambert, D. L., \& Allende Prieto, C. 2002, MNRAS, 335, 325

8. Mashonkina, L., \& Zhao, G. 2006, A\&A, 456, 313

9. Sneden, C., Cowan, J. J., Lawler, J. E., Burles, S., Beers, T. C., \& Fuller, G. M. 2002, ApJ, 566, L25

10. Aoki, W., Honda, S., Beers, T. C., \& Sneden, C. 2003a, ApJ, 586, 506

11. Aoki, W., et al. 2003b, ApJ, 592, L67

12. Lundqvist, M., Wahlgren, G. M., \& Hill, V. 2007, A\&A, 463,693

13. Lawler, J. E., Wickliffe, M. E., den Hartog, E. A., \& Sneden, C. 2001, ApJ, 563, 1075

14. Ivans, I. I., Simmerer, J., Sneden, C., Lawler, J. E., Cowan, J. J., Gallino, R., \& Bisterzo, S. 2006, ApJ, 645, 613

15. Den Hartog, E. A., Lawler, J. E., Sneden, C., \& Cowan, J. J. 2003, ApJS, 148, 543

16. Rosner, S. D., Masterman, D., Scholl, T. J., \& Holt, R. A. 2005, Can. J. Phys., 83, 841

17. Masterman, D., Rosner, S. D., Scholl, T. J., Sharikova, A., \& Holt, R. A. 2003, Can. J. Phys., 81, 1389

18. Lawler, J. E., Den Hartog, E. A., Sneden, C., \& Cowan, J. J. 2006, ApJS, 162, 227

19. Sneden, C. A. 1973, Ph.D. Thesis, Univ. Texas at Austin

20. Learner, R. C. M., \& Thorne, A. P. 1988, J. Opt. Soc. Am. $\mathrm{B}, 5,2045$

21. Nave, G., Johansson, S., Learner, R. C. M., Thorne, A. P., \& Brault, J. W. 1994, ApJS, 94, 221

22. Pickering, J. C., Thorne, A. P., \& Perez, R. 2001, ApJS, 132,403

23. Pickering, J. C., Thorne, A. P., \& Perez, R. 2002, ApJS, 138,247

24. Lucatello, S., Tsangarides, S., Beers, T. C., Carretta, E., Gratton, R. G., \& Ryan, S. G. 2005, ApJ, 625, 825 